\title{
Comparative optical study of colloidal anatase titania nanorods and atomically thin wires
}

Received 16th November 2012

Andrei S. Susha, ${ }^{a}$ Andrey A. Lutich, ${ }^{\mathrm{b}}$ Chenmin Liu, ${ }^{\mathrm{c}} \mathrm{Hu} \mathrm{Xu},{ }^{\mathrm{a}}$ Ruiqing Zhang, ${ }^{\mathrm{a}}$ Yongchun Zhong, ${ }^{d}$ Kam Sing Wong, ${ }^{d}$ Shihe Yang ${ }^{c}$ and Andrey L. Rogach ${ }^{* a}$

Accepted 2nd January 2013

DOI: $10.1039 / c 2 n r 33669 c$

www.rsc.org/nanoscale

We present results of a comparative study of colloidal anatase titanium oxide nanorods and extremely thin atomic wires of systematically decreasing ( $2.6 \mathrm{~nm}$ down to $0.5 \mathrm{~nm}$ ) diameter in terms of their optical absorption as well as steady-state and time-resolved photoluminescence. Steady-state photoluminescence spectra of the titania samples show three well-distinguished spectral components, which are ascribed to excitonic emission $(4.26 \pm 0.2 \mathrm{eV})$, as well as radiative recombination of trapped holes with electrons from the conduction band (4.04 $\pm 0.4 \mathrm{eV})$ and radiative recombination of trapped electrons with holes in the valence band $(3.50 \pm 0.2 \mathrm{eV})$ in nanocrystalline anatase $\mathrm{TiO}_{2}$. Time-resolved photoluminescence measurements point out the existence of different emissive species responsible for the appearance of high-energetic and low-energetic emission peaks of $\mathrm{TiO}_{2}$ atomic wires and nanorods.

Titanium dioxide is an extremely popular, environmentally benign and low cost material with a variety of potential applications ranging from water splitting ${ }^{1}$ and sodium ion batteries ${ }^{2}$ to photoelectrochemical solar cells, ${ }^{3,4}$ to name a few. Synthesis, properties and applications of nanostructured $\mathrm{TiO}_{2}$ have been a focus of extensive research in the last two decades, ${ }^{5}$ with a particular emphasis on the charge recombination and transport. ${ }^{6,7}$ Recent advances of physical chemistry of $\mathrm{TiO}_{2}$ nanostructures and their perspectives for photocatalysis and photovoltaics have been summarized in a recent Editorial by Kamat. $^{8}$ The anatase phase is stabilized over rutile in

${ }^{a}$ Department of Physics and Materials Science \& Centre for Functional Photonics (CFP), City University of Hong Kong, Tat Chee Avenue, Kowloon, Hong Kong S.A.R. E-mail: andrey.rogach@cityu.edu.hk

${ }^{b}$ Photonics and Optoelectronics Group, Physics Department and Center for NanoScience (CeNS), Ludwig-Maximilians-Universität München, Amalienstr. 54, D-80799 Munich, Germany

${ }^{c}$ Department of Chemistry \& William Mong Institute of Nano Science and Technology, The Hong Kong University of Science and Technology, Clear Water Bay, Kowloon, Hong Kong S.A.R.

${ }^{d}$ Department of Physics, The Hong Kong University of Science and Technology, Clear Water Bay, Kowloon, Hong Kong S.A.R. nanocrystalline $\mathrm{TiO}_{2},{ }^{9}$ and there have been continuous efforts in developing synthetic routes leading to high-quality anatase $\mathrm{TiO}_{2}$ nanoparticles of small sizes ${ }^{\mathbf{1 0 , 1 1}}$ and controllable dimensions, in particular rod-shaped nanocrystals. ${ }^{\mathbf{1 2 , 1 3}}$ The available palette of colloidally synthesized, anisotropic $\mathrm{TiO}_{2}$ nanostructures has been recently extended towards extremely thin anatase wires with diameters reaching the atomic limit of a few angstroms. ${ }^{\mathbf{1 4 , 1 5}}$ Size and doping effects on the absorption and emission properties of colloidal $\mathrm{TiO}_{2}$ nanoparticles have been studied in detail by Serpone and co-workers, ${ }^{16,17}$ and have been interpreted in terms of the co-existence of the band-edge ${ }^{18}$ (or self-trapped exciton ${ }^{19}$ ) recombination and the recombination involving midgap energy levels originating from oxygen vacancies (F-type color centres). ${ }^{20,21}$ Recent studies by Knorr, McHale and co-workers ${ }^{22-24}$ addressed the origin of the trap-related emission in nanocrystalline $\mathrm{TiO}_{2}$, classifying it into contributions from two spatially isolated trap-state distributions, resulting in recombination of trapped electrons or holes with oppositely charged mobile charge carriers in the valence or conduction band, respectively. Yoshihara et al. employed transient absorption spectroscopy to study the localization of trapped electrons and holes in nanocrystalline $\mathrm{TiO}_{2}$, which have been found to be at the surface of the particle while free electrons were distributed in the bulk. ${ }^{25}$ It has been pointed out that the trap-state distribution for $\mathrm{TiO}_{2}$ samples strongly depends on their preparation and history, so that both the energetic distribution of traps and the positions of the respective photoluminescence (PL) maxima vary strongly in literature reports. At the same time, the manifestation of the size quantization effect influencing the photophysical properties of colloidal anatase $\mathrm{TiO}_{2}$ nanoparticles has remained controversial over decades. ${ }^{16,20}$ Taking advantage of the availability of the colloidal anatase $\mathrm{TiO}_{2}$ nanorods with a diameter systematically decreasing towards the dimensions of extremely thin atomic wires, which can be synthesized within one and the same nonhydrolytic solution approach by variation of the reaction time, ${ }^{15}$ we have conducted a systematic study of their optical absorption as well as steady-state and time-resolved PL, with the results presented and discussed here. 
Anatase $\mathrm{TiO}_{2}$ nanorods and atomic wires, which are denoted further in the text according to their dimensions (diameter $\times$ length) as $2.6 \times 20 \mathrm{~nm}$ nanorods and $0.5 \times 20 \mathrm{~nm}$ atomic wires, have been synthesized starting from the same titanium complex precursor. The latter was obtained by mixing $3 \mathrm{~mL}$ of oleic acid with $10 \mathrm{~mL}$ of cyclohexane $(10 \mathrm{~mL})$, followed by dropwise addition of $0.5 \mathrm{~mL}$ of $\mathrm{Ti}(\mathrm{OBu})_{4}$. The mixture was sealed in a Teflon-lined stainless autoclave, heated to $150{ }^{\circ} \mathrm{C}$ and kept at that temperature for $25 \mathrm{~h}$, yielding a light-yellow but transparent, viscous liquid which was then extracted by precipitation with an excess of ethanol at room temperature, and re-dispersed in a mixture of 1-octadecene (ODE, $5 \mathrm{~mL}$ ), oleic acid $(0.6 \mathrm{~mL})$, and oleylamine (OLA, $0.8 \mathrm{~mL}$ ). To grow $2.6 \times 20 \mathrm{~nm}$ nanorods, this solution was heated to and maintained at $300{ }^{\circ} \mathrm{C}$ in a threenecked flask with stirring for $2 \mathrm{~h}$. To grow $0.5 \times 20 \mathrm{~nm}$ atomic wires, the solution was heated to and maintained at $180{ }^{\circ} \mathrm{C}$ in a three-necked flask with stirring for $1 \mathrm{~h}$. Yet another anatase $\mathrm{TiO}_{2}$ nanorod sample, denoted as $1.8 \times 40 \mathrm{~nm}$ nanorods further in the text, has been prepared by a one-step variant of the abovedescribed method, where the mixture of oleic acid $(3 \mathrm{~mL})$, cyclohexane $(10 \mathrm{~mL})$, and $\mathrm{Ti}(\mathrm{OBu})_{4}(0.1 \mathrm{~mL})$ was stirred at room temperature for $1 \mathrm{~h}$, followed by a dropwise addition of $1 \mathrm{~mL}$ of oleylamine. The resulting solution was sealed in a Teflon-lined stainless autoclave, heated to $150{ }^{\circ} \mathrm{C}$, and kept for $25 \mathrm{~h}$. Both nanorods and atomic wires were precipitated by excess of ethanol, and purified by two circles of centrifugation and washing with ethanol. The final products were easily redispersible in solvents such as chloroform or hexane without any sign of further growth or irreversible aggregation. Fig. 1 shows TEM overview images of the three samples, with insets providing HRTEM images of the respective representative nanorods and nanowires. All nanostructures are reasonably monodisperse both in terms of their diameter and length, and remain well separated even after the deposition onto TEM grids due to the presence of organic surfactants. The dimensions of 3 samples have been estimated based on the statistical analysis of HRTEM images of the respective samples, as has been discussed in detail in ref. 15 .

All optical absorption measurements were performed on highly diluted solutions of $\mathrm{TiO}_{2}$ nanorods/nanowires in the same solvent chloroform without any sign of aggregation which may be accounting for undesirable scattering effects. Both integrated and time-resolved PL measurements were performed on diluted colloidal solutions having an optical density below 0.2 at the respective excitation wavelengths. Absorption spectra of the three titania samples (Fig. 2) show a pronounced maximum centered at $4.55 \mathrm{eV}$, consisting of several well resolved peaks, and a few shoulders at lower energies $(3.9 \mathrm{eV}$ and $4.35 \mathrm{eV}$ ). The signatures of multiple electronic transitions in the absorption spectra of anatase $\mathrm{TiO}_{2}$ nanoparticles have been reported previously ${ }^{16}$ and have been assigned to direct electronic transitions in an otherwise indirect bandgap $\mathrm{TiO}_{2}$ bulk semiconductor by the comparison with its calculated electronic band structure according to Daude et al. ${ }^{26}$ Our own calculations of the electronic structure of $\mathrm{TiO}_{2}$ atomic wires conducted using the density functional theory within the generalized gradient approximation as implemented in the
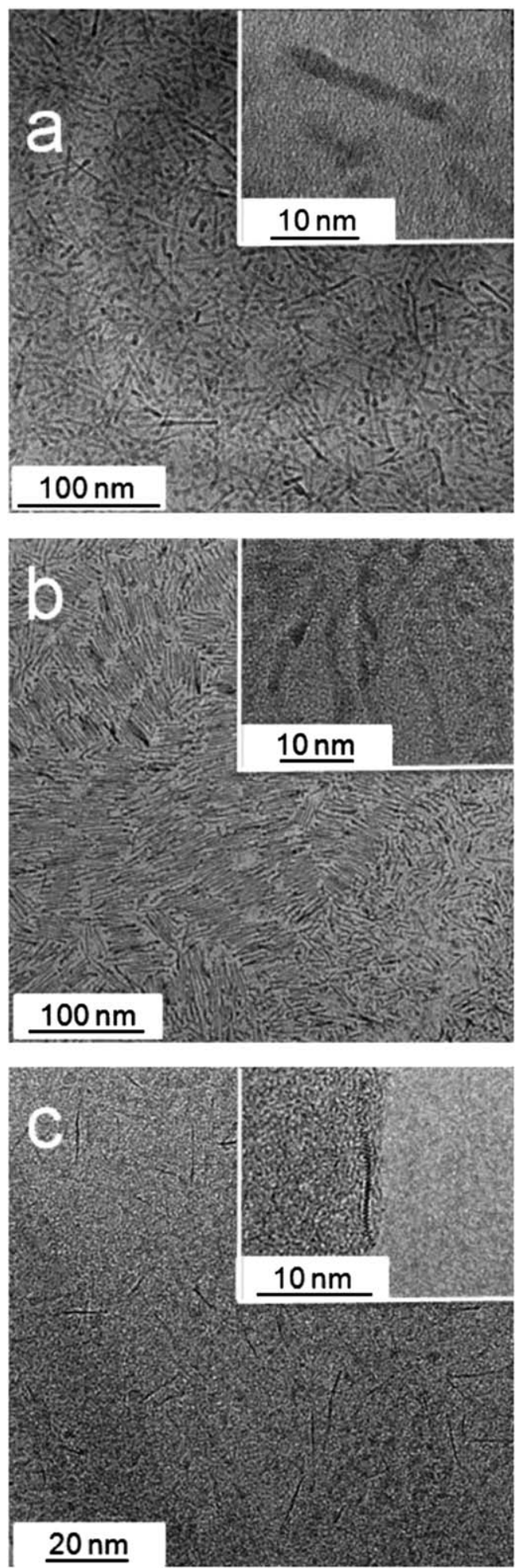

Fig. 1 TEM images of $\mathrm{TiO}_{2}$ (a) $2.6 \times 20 \mathrm{~nm}$ nanorods, (b) $1.8 \times 40 \mathrm{~nm}$ nanorods, and (c) $0.5 \times 20 \mathrm{~nm}$ atomic wires. Insets provide HRTEM images of the respective representative samples.

Vienna $a b$ initio simulation package $\mathrm{e}^{27}$ confirm that the atomic wires exhibit a direct energy band gap, with several electronic transitions at $4.39 \mathrm{eV}, 4.35 \mathrm{eV}, 4.62 \mathrm{eV}$ and $4.74 \mathrm{eV}$. They further reveal that the bandgap of atomic wires broadens as compared 

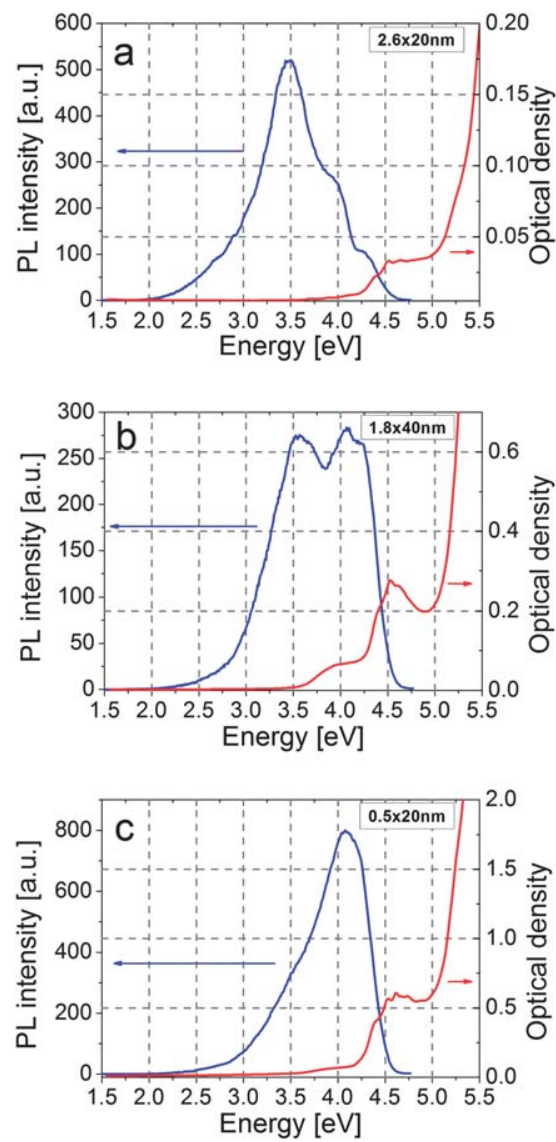

Fig. 2 Absorption (red) and PL (blue) spectra of $\mathrm{TiO}_{2} 2.6 \times 20 \mathrm{~nm}$ nanorods, $1.8 \times 40 \mathrm{~nm}$ nanorods, and $0.5 \times 20 \mathrm{~nm}$ atomic wires. The excitation energy was $4.96 \mathrm{eV}$ for all PL spectra.

to bulk anatase $\mathrm{TiO}_{2}$, in good agreement with previous report. ${ }^{28}$ We note that all the experimentally measured absorption features are strongly blue-shifted from the band gap energy value for bulk anatase $\mathrm{TiO}_{2}(3.2 \mathrm{eV})^{17}$ but do not differ much in the size regime of the three samples studied here. This is consistent with previously reported data of Serpone et al. ${ }^{16}$ related to the manifestation of size quantization effects in $\mathrm{TiO}_{2}$ nanosized colloids.

The steady-state PL spectra of all three titania samples reproducibly show three well-distinguished spectral components, manifesting themselves as peaks or shoulders centered at $4.26 \pm 0.2 \mathrm{eV}, 4.04 \pm 0.4 \mathrm{eV}$ and $3.50 \pm 0.2 \mathrm{eV}$, as illustrated in Fig. 2. The relative PL intensities of these three components change systematically upon the diameter of the quasi-one dimensional titania: $2.6 \times 20 \mathrm{~nm}$ nanorods show a pronounced low-energetic peak and two shoulders at higher energy; thinner $1.8 \times 40 \mathrm{~nm}$ nanorods show PL peaks of almost equal intensities, while for the $0.5 \times 20 \mathrm{~nm}$ atomic wires the high-energy PL peak, superimposed with the second spectral component, becomes dominant. PL measurements point to recombination processes controlled by near surface atoms in particular in $\mathrm{TiO}_{2}$ nanowires. Based on the literature data, the observed three PL maxima could be ascribed to (i) excitonic emission (4.26 \pm $0.2 \mathrm{eV}$ ), ${ }^{18}$ as well as (ii) radiative recombination of trapped holes with electrons from the conduction band $(4.04 \pm 0.4 \mathrm{eV})$, and (iii) radiative recombination of trapped electrons with holes in the valence band ${ }^{23,24}(3.50 \pm 0.2 \mathrm{eV})$ in nanocrystalline anatase $\mathrm{TiO}_{2}$. The latter two types of emission in nanocrystalline $\mathrm{TiO}_{2}$ originate from the distribution of surface traps ${ }^{25}$ associated with oxygen vacancies ${ }^{16,18,29,30}$ and typically manifest themselves as "green" and "red" emission in bulkier $\mathrm{TiO}_{2}$ samples studied in ref. 22-24, while for the smaller colloidal titania nanoparticles PL maxima have been reported to shift towards higher energies. ${ }^{16,18}$ The energetic distribution of in particular electron trap states located about $0.7 \mathrm{eV}$ above the valence band edge (Fig. 2) coincides well with the previously published data. ${ }^{23}$ Same as for the absorption edge, there are no pronounced shifts in the spectral positions of the PL maxima of the excitonic transition of $\mathrm{TiO}_{2}$ samples studied here depending on their diameters, even so all the spectra are strongly blue-shifted from the position of bulk anatase titania bandgap.

Room temperature PL quantum yields (QY) of the three $\mathrm{TiO}_{2}$ samples have been estimated by comparison to anthracene dissolved in ethanol, with a known PL QY of $27 \%,{ }^{31}$ and are summarized in Table 1. The increase of QY goes hand in hand with the decreasing surface-to-volume ratio for the increasing rod/wire diameter (Table 1). We have further measured PL decays of the three $\mathrm{TiO}_{2}$ samples using a Hamamatsu model C4334 streak camera coupled to a spectrometer. The third harmonic of the laser output at $267 \mathrm{~nm}(4.64 \mathrm{eV})$ from a femtosecond titanium-sapphire oscillator was used as the excitation source, with a pulse width and a repetition rate of $200 \mathrm{fs}$ and $76 \mathrm{MHz}$, respectively. The excitation power was $0.1 \mathrm{~mW}$; the integration window was $4 \mathrm{~nm}$ and the center of the window was set at the position of the exciton emission peak/ shoulder at $4.26 \mathrm{eV}$ as well as at positions of the higher $(4.05 \mathrm{eV}) /$ lower (3.55) energetic trap emission peak as they appear in Fig. 2. A two-exponential decay function had been used to fit the PL decay curves to derive fast and long components $\tau_{1}$ and $\tau_{2}$ for the three $\mathrm{TiO}_{2}$ samples, which are summarised in Table 1. The representative PL decay curves detected at the position of the $4.05 \mathrm{eV}$ PL peak are shown in Fig. 3. When comparing the PL decays for three different PL peaks, it appears that the $4.05 \mathrm{eV}$ PL peak has the slowest PL decay, while $4.26 \mathrm{eV}$ and $3.55 \mathrm{eV}$ peaks have relatively fast decays (Table 1 ). In general, the larger the diameter of the rod/wire, the shorter the PL lifetime for both components of PL decays (Fig. 3 and Table 1), which further points out that the high- and the low-energetic emission peaks belong to different emitting species.

In conclusion, the results of a comparative optical study of colloidal anatase titanium oxide nanorods and extremely thin atomic wires of systematically decreasing $(2.6 \mathrm{~nm}, 1.8 \mathrm{~nm}$ and $0.5 \mathrm{~nm}$ ) diameter, produced under identical synthetic conditions, point out several interesting observations. Both for the absorption edge and for the emission maxima of the three samples, there are no pronounced shifts in the spectral positions upon the change in their diameter, even so they are strongly blue-shifted from the position of the bulk anatase titania bandgap, as is predicted by theoretical calculations. The steady-state photoluminescence spectra of the titania samples show three well-distinguished spectral components, which are 


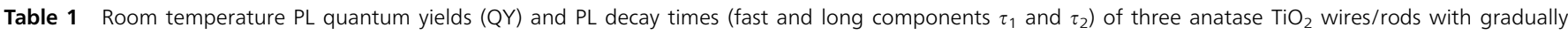
increasing diameters. All PL decays have been measured with an excitation energy of $4.64 \mathrm{eV}$, and detected at positions of three different PL peaks indicated

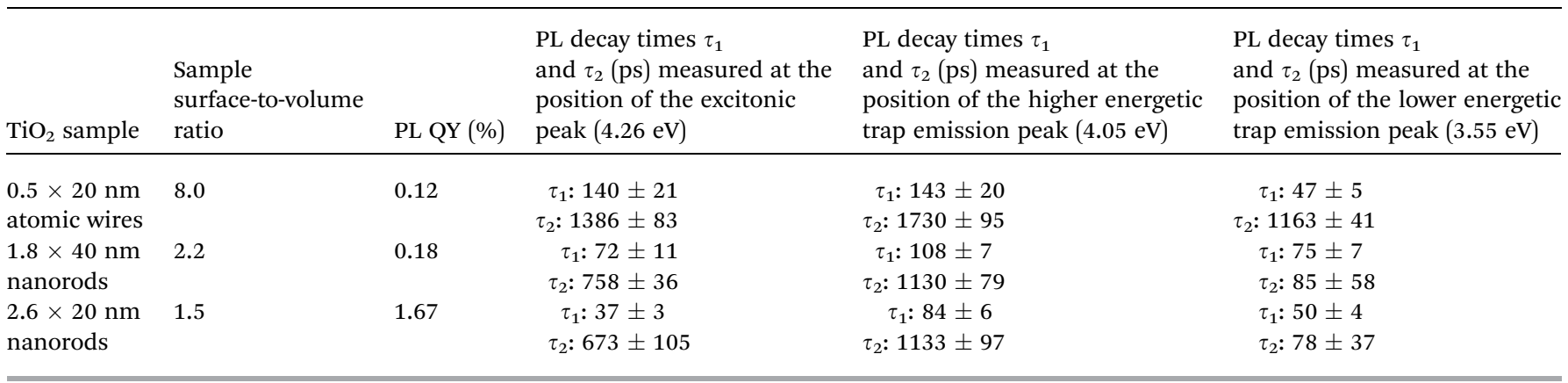

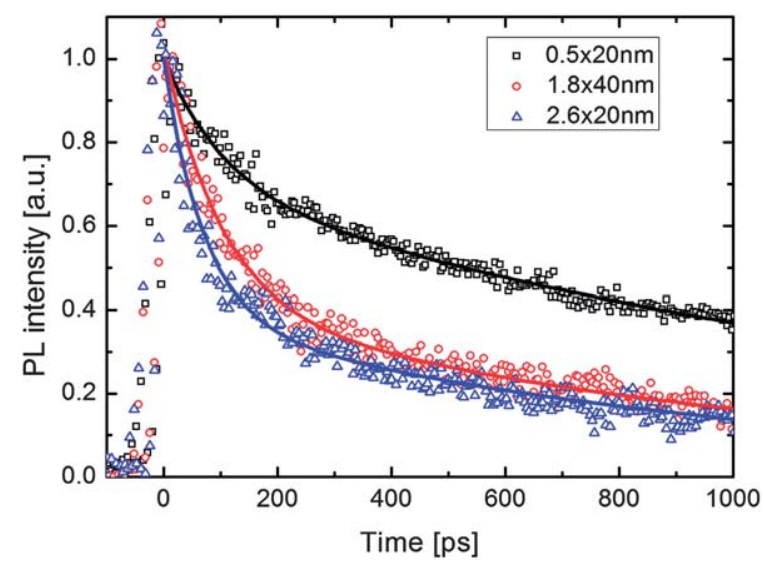

Fig. 3 Normalized PL decays spectra of three different $\mathrm{TiO}_{2}$ samples, indicated on the frame. The excitation energy was $4.64 \mathrm{eV}$ for all spectra, and the detection was done at the position of the higher energetic trap emission peak $(4.05 \mathrm{eV})$. Solid lines represent the double exponential fits to experimental data.

ascribed to excitonic emission $(4.26 \pm 0.2 \mathrm{eV})$, as well as radiative recombination of trapped holes with electrons from the conduction band $(4.04 \pm 0.4 \mathrm{eV})$ and radiative recombination of trapped electrons with holes in the valence band $(3.50 \pm 0.2 \mathrm{eV})$ in nanocrystalline anatase $\mathrm{TiO}_{2}$. Time-resolved photoluminescence measurements further point out the existence of different emissive species responsible for the appearance of high-energetic and low-energetic emission peaks of $\mathrm{TiO}_{2}$ atomic wires and nanorods.

\section{Acknowledgements}

The authors acknowledge financial support from the Research Grant Council of Hong Kong S.A.R. (projects no. T23-713/11, RGC-GRF HKUST605710 and HKUST2/CRF/11G), from the DFG through the Nanosystems Initiative Munich (NIM), and from the Centre of Functional Photonics of City University of Hong Kong.

\section{References}

1 A. Fujishima and K. Honda, Nature, 1972, 238, 37.
2 H. Xiong, M. D. Slater, M. Balasubramanian, C. S. Johnson and T. Rajh, J. Phys. Chem. Lett., 2011, 2, 2560.

3 M. Gratzel, Nature, 2001, 414, 338.

4 D. H. Chen, F. Z. Huang, Y. B. Cheng and R. A. Caruso, Adv. Mater., 2009, 21, 2206.

5 X. Chen and S. S. Mao, Chem. Rev., 2007, 107, 2891.

6 M. Bailes, P. J. Cameron, K. Lobato and L. M. Peter, J. Phys. Chem. B, 2005, 109, 15429.

7 N. M. Dimitrijevic, Z. V. Saponjic, B. M. Rabatic, O. G. Poluektov and T. Rajh, J. Phys. Chem. C, 2007, 111, 14597.

8 P. V. Kamat, J. Phys. Chem. C, 2012, 116, 11849.

9 H. Z. Zhang and J. F. Banfield, J. Phys. Chem. B, 2000, 104, 3481.

10 M. Niederberger, M. H. Bartl and G. D. Stucky, Chem. Mater., 2002, 14, 4364.

11 X. L. Li, Q. Peng, J. X. Yi, X. Wang and Y. D. Li, Chem.-Eur. J., 2006, 12, 2383.

12 P. D. Cozzoli, A. Kornowski and H. Weller, J. Am. Chem. Soc., 2003, 125, 14539.

13 Z. H. Zhang, X. H. Zhong, S. H. Liu, D. F. Li and M. Y. Han, Angew. Chem., Int. Ed., 2005, 44, 3466.

14 C. M. Liu and S. H. Yang, ACS Nano, 2009, 3, 1025.

15 C. M. Liu, H. Sun and S. H. Yang, Chem.-Eur. J., 2010, 16, 4381.

16 N. Serpone, D. Lawless and R. Khairutdinov, J. Phys. Chem., 1995, 99, 16646.

17 N. Serpone, J. Phys. Chem. B, 2006, 110, 24287.

18 N. D. Abazovic, M. I. Comor, M. D. Dramicanin, D. J. Jovanovic, S. P. Ahrenkiel and J. M. Nedeljkovic, J. Phys. Chem. B, 2006, 110, 25366.

19 J. Preclikova, P. Galar, F. Trojanek, S. Danis, B. Rezek, I. Gregora, Y. Nemcova and P. Maly, J. Appl. Phys., 2010, 108, 113502.

20 V. N. Kuznetsov and N. Serpone, J. Phys. Chem. B, 2006, 110, 25203.

21 V. N. Kuznetsov and N. Serpone, J. Phys. Chem. C, 2009, 113, 15110.

22 F. J. Knorr, D. Zhang and J. L. McHale, Langmuir, 2007, 23, 8686.

23 F. J. Knorr, C. C. Mercado and J. L. McHale, J. Phys. Chem. C, 2008, 112, 12786. 
24 C. C. Mercado, F. J. Knorr, J. L. McHale, S. M. Usmani, A. S. Ichimura and L. V. Saraf, J. Phys. Chem. C, 2012, 116, 10796.

25 T. Yoshihara, R. Katoh, A. Furube, Y. Tamaki, M. Murai, K. Hara, S. Murata, H. Arakawa and M. Tachiya, J. Phys. Chem. B, 2004, 108, 3817.

26 N. Daude, C. Gout and C. Jouanin, Phys. Rev. B: Solid State, 1977, 15, 3229.
27 G. Kresse and J. Furthmuller, Phys. Rev. B: Condens. Matter Mater. Phys., 1996, 54, 11169.

28 D. Tafen and J. P. Lewis, Phys. Rev. B: Condens. Matter Mater. Phys., 2009, 80, 014104.

29 T. Dittrich, Phys. Status Solidi A, 2000, 182, 447.

30 G. K. Boschloo and A. Goossens, J. Phys. Chem., 1996, 100, 19489.

31 W. H. Melhuish, J. Phys. Chem., 1961, 65, 229. 